\title{
Developmental immunotoxicology: What underlies the critical windows of exposure?
}

Autor:

Palabras clave:
Moreira, C.; Paiola, Matthieu; Duflot, A.; Pozo, R. del; Piazzon de Haro, María Carla ; Fouz, B.; Varó, Inmaculada CSIC; Sitjà-Bobadilla, Ariadna CSIC ; Knigge, Thomas; Pinto, P.; Monsinjon, Tiphaine

Developmental immunotoxicity

Immune system

Critical windows

Endocrine disruptors

Oestrogens

Fecha de publicación:

17 -jun-2019 
Endocrine disruptors in general and oestrogenic compounds in particular have been widely investigated in view of their effects on several physiological processes and, therefore, their ecotoxicologic relevance. The modulation of the immune system by oestrogens has increasingly sparked interest in the research community, that previously had been mainly centered on the reproductive effects of these hormones. In fact, since the industrialization an increasing variety of endocrine disruptors, such as oestrogenic endocrine disruptors, are retrieved in the environment. These oestrogenic endocrine disrupting chemicals (EEDCs) have also been suggested to increase the prevalence of autoimmune diseases and cancer. With regard to the high degree of similarities in the immune system of jawed vertebrates as well as the conserved immunomodulatory roles of oestrogen, environmental EEDCs possibly have the capacity to alter immune system functions of teleost fish, which may impair their capacity to fight infectious diseases and eventually may contribute, together with overfishing, to wild stock losses. Importantly, the most deleterious effects of EEDCs, both in mammals and teleosts, appear to arise when the exposure occurs during specific periods of the immune system ontogenesis, commonly referred as critical windows of exposure. However, in mammals and especially in teleost fish, these stages of the immune system development as well as the EEDC-action remain to be fully identified and characterised. The concept and the importance of developmental immunotoxicity is presented by addressing the mechanisms of oestrogenic regulation and the mode of action of EEDCs from an immunological perspective. Emphasis is given to the critical windows of development of the immune system during which EEDCs may alter the immune system development and function with long-term consequences on immunocompetence. Results from different classes of vertebrates are compiled, highlighting studies on teleost fish and their relevance for the human immune system. Additionally, new results on the effects of environmentally relevant concentration of exogenous estradiol exposure during European sea bass (Dicentrarchus labrax) development will be presented with regard to oestrogen's ability to trigger effects on 
immunocompetence, contributing to fill the gaps on vertebrate immunotoxicology. 